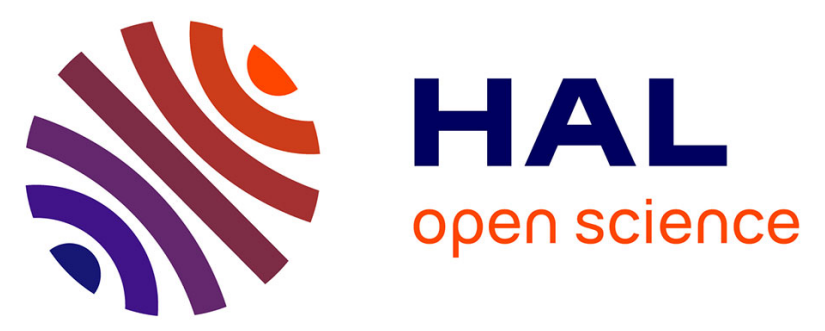

\title{
Single-Frequency Operation of a Near-degenerate Optical Parametric Oscillator Using a Transversally Chirped Volume Bragg Grating
}

Adeline Kabacinski, Julie Armougom, Jean-Michel Melkonian, Myriam Raybaut, Jean-Baptiste Dherbecourt, Antoine Godard, Ruslan Vasilyeu, Vadim Smirnov

\section{To cite this version:}

Adeline Kabacinski, Julie Armougom, Jean-Michel Melkonian, Myriam Raybaut, Jean-Baptiste Dherbecourt, et al.. Single-Frequency Operation of a Near-degenerate Optical Parametric Oscillator Using a Transversally Chirped Volume Bragg Grating. CLEO 2019, May 2019, SAN JOSE, United States. 10.1364/CLEO_SI.2019.SF1H.3 . hal-02322892

\section{HAL Id: hal-02322892 \\ https://hal.science/hal-02322892}

Submitted on 21 Oct 2019

HAL is a multi-disciplinary open access archive for the deposit and dissemination of scientific research documents, whether they are published or not. The documents may come from teaching and research institutions in France or abroad, or from public or private research centers.
L'archive ouverte pluridisciplinaire HAL, est destinée au dépôt et à la diffusion de documents scientifiques de niveau recherche, publiés ou non, émanant des établissements d'enseignement et de recherche français ou étrangers, des laboratoires publics ou privés. 


\title{
Single-Frequency Operation of a Near-degenerate Optical Parametric Oscillator Using a Transversally Chirped Volume Bragg Grating
}

\author{
Adeline Kabacinski ${ }^{1}$, Julie Armougom ${ }^{1}$, Jean-Michel Melkonian ${ }^{1 *}$, Myriam Raybaut ${ }^{1}$, Jean-Baptiste \\ Dherbecourt $^{1}$, Antoine Godard ${ }^{1}$, Ruslan Vasilyeu ${ }^{2}$, Vadim Smirnov ${ }^{2}$ \\ ${ }^{1}$ DPHY, ONERA, Université Paris Saclay, F-91123 Palaiseau, France \\ ${ }^{2}$ OptiGrate Corp (an IPG Photonics Company), 562 S Econ Cir, Oviedo, FL 32765, USA \\ *jean-michel.melkonian@onera.fr
}

\begin{abstract}
We report on single-frequency emission near $2.2 \mu \mathrm{m}$ with an optical parametric oscillator. The OPO wavelength is tuned over $22 \mathrm{~nm}$ using a Bragg reflector incorporating a transverse chirp of the grating period. (C) 2019 The Author(s)

OCIS codes: (190.4970) Parametric oscillators and amplifiers; (230.1480) Bragg reflectors.
\end{abstract}

\section{Introduction}

Spectral selection in an Optical Parametric Oscillator (OPO) behaves very differently than in a laser. It is essentially driven by the phase difference between the generated signal and idler waves and the nonlinear polarization created by the pump. This so-called phase-matching condition writes, in the collinear case: $n_{p} \sigma_{p}-n_{s} \sigma_{s}-n_{i} \sigma_{i}<$ $\Delta \phi_{\max } / 2 \pi L$, where $\mathrm{n}_{\mathrm{p}, \mathrm{s}, \mathrm{i}}$ are the index of refraction at wavenumbers $\sigma_{\mathrm{p}, \mathrm{s}, \mathrm{i}}, L$ the crystal length, and $\Delta \phi_{\max }$ is the maximum dephasing allowed by the parametric gain. Derivation of this relation leads to the total first-order gain bandwidth:

$$
2 \Delta \sigma_{s}=\frac{1}{\pi L} \frac{\Delta \phi_{\max }}{n_{g, i}-n_{g, s}},
$$

where $n_{\mathrm{g}}$ is the group velocity index. Under pulsed pumping, several longitudinal modes oscillate within the gain bandwidth and, since the oscillation starts from parametric noise, these modes will differ from one pulse to another. This problem is the worst in OPOs using near-degenerate signal and idler waves $\left(\sigma_{s}=\sigma_{i}=\sigma_{p} / 2\right)$ with identical polarizations (type 0 or type I phase-matching), because in this case $n_{g, s}=n_{g, i}$, so that the gain bandwidth becomes very broad. We circumvented this issue by introducing Vernier spectral filtering in doubly-resonant cavities, in which spectral selection is achieved by the coincidence between signal and idler cavity modes [1]. For this scheme to be still effective near degeneracy, we had to resort to periodically-poled lithium niobate (PPLN) with crossed polarizations such that $n_{g, s} \neq n_{g, i}$ (type II phase-matching) [2]. The low non-linear coefficient of PPLN with crossed polarizations was partially balanced by the very low threshold offered by the doubly-resonant cavity.

Recently, Volume Bragg Gratings (VBGs) have emerged as very-narrow, high-efficiency spectral filters for laser applications. They have been used in OPOs to provide narrowband tunable emission [3], and single-frequency operation at a fixed wavelength off degeneracy [4]. In this work, we use a VBG to produce tunable single-frequency radiation very close to degeneracy, while benefiting from the high gain of PPLN with identical polarizations.

\section{Design of the transversally chirped Volume Bragg Grating}

VBGs fabricated in photo-thermo-refractive (PTR) glass have outstanding properties in the near infrared providing extremely high spectral and angular selectivity with no secondary peak, and diffraction efficiency up to 99.99\% [5]. Unlike other holographic materials, PTR glass-based elements have robust performance in harsh environmental condition, unrestricted life time and high laser damage threshold. All above pave the way for their usage as intracavity mode filters in various laser systems.

A transversally chirped volume Bragg grating (TC-BG) is a reflecting VBG in which the transverse period varies across one lateral dimension. Nowadays, state-of-the-art technology allows fabrication of such gratings with lateral dimensions up to $25 \mathrm{~mm}$. For this experiment, we designed a TC-BG which is $20 \mathrm{~mm}$ wide, with a chirp rate of $1.1 \mathrm{~nm} / \mathrm{mm}$. The FWHM spectral acceptance is $1.2 \mathrm{~nm}$ around $2.2 \mu \mathrm{m}$ and the maximum diffraction efficiency is $80 \%$. The TC-BG is AR-coated at $1.064 \mu \mathrm{m}+2-2.2 \mu \mathrm{m}$. The angular acceptance was checked to be much larger than the mode divergence inside the cavity. 


\section{Experimental results}

The OPO is doubly-resonant with nested signal and idler cavities (Fig. 1, left). The non-linear crystal is a 4.2-mmlong MgO:PPLN crystal with a quasi-phase-matching period of $32.03 \mu \mathrm{m}$ for identical pump, signal, and idler polarizations. The two-pass first-order gain bandwidth given by Eq. 1 is $724 \mathrm{~cm}^{-1}$ (spanning 1800 longitudinal modes). The signal cavity is formed by a gold mirror with a $50 \mathrm{~mm}$ radius of curvature $\left(\mathrm{M}_{3}\right)$ and a highly reflective dielectric mirror deposited on the input crystal facet $\left(\mathrm{M}_{2}\right)$. The idler cavity is formed by the gold mirror and the TCBG which acts as a plane output coupler. The TC-BG is mounted on a transverse translation stage to shift the peak wavelength of the reflectivity curve. The distance between the TC-BG and the signal mirror has been chosen to allow only one signal/idler mode coincidence within the TC-BG bandwidth (Fig.1, right). The TC-BG and the back mirror are mounted on PZT actuators to match the signal and idler combs and finely tune the output wavelength. The oscillation threshold with the TC-BG is $7.5 \mu \mathrm{J}$, identical to the one obtained with a dielectric mirror of the same reflectivity.
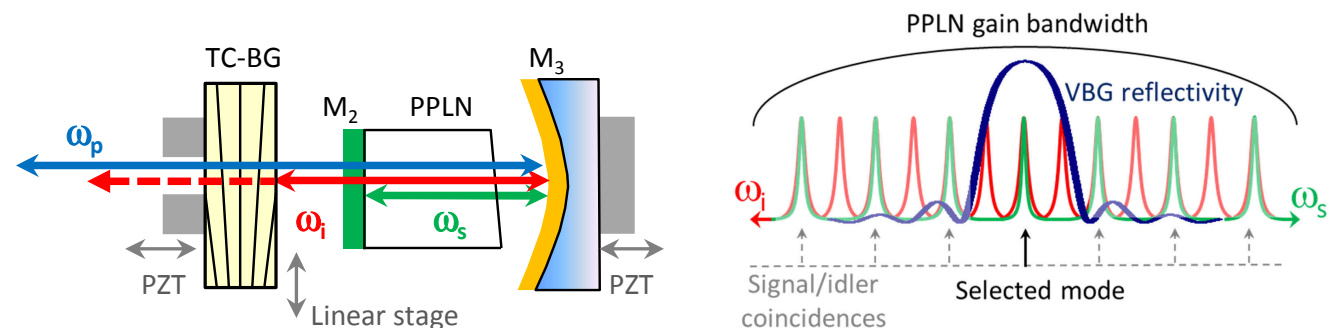

Fig. 1. Left: Scheme of the nested-cavity OPO cavity with the TC-BG. Right: Spectral filtering is a combination of signal/idler combs coincidence and selection by the reflectivity of the TC-BG.

Fig. 2 shows the emission spectrum of the OPO when translating the TC-BG, and tuning the PZT actuators to maximize the signal/idler mode overlap. A total tunability of $17 \mathrm{~nm}$ was achieved, compliant with the TC-BG specifications. As observed with our previous nested-cavity OPOs, several modes oscillate when the signal and idler combs are mismatched. What is remarkable here is that, despite the huge gain bandwidth, only two neighboring modes are allowed to oscillate, thanks to the high selectivity of the TC-BG. When the signal and idler combs are correctly matched using the PZTs, the OPO emits single-frequency radiation.
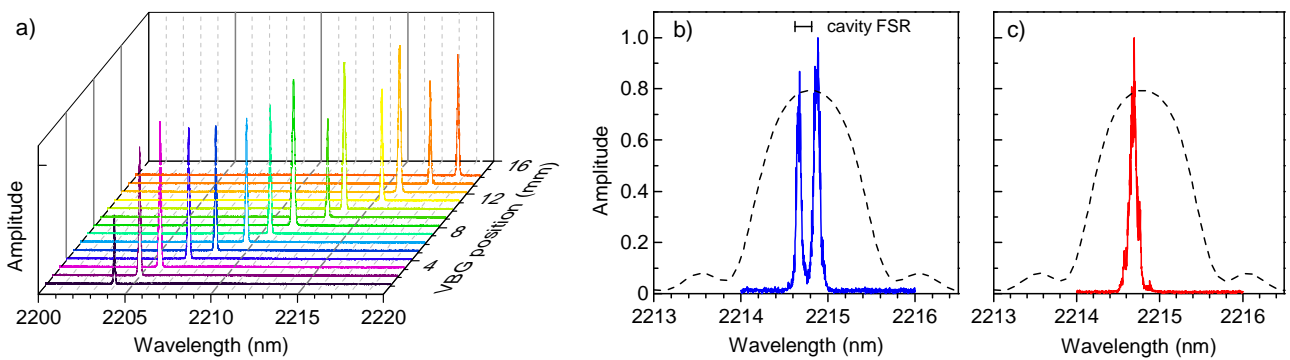

Fig. 2. a) Spectrum at different TC-BG positions. Spectrum at one TC-BG position for b) mismatched and c) matched signal/idler combs. The peak width is limited by the spectrometer resolution. The black dashed line is the calculated reflectivity curve of the TC-BG.

\section{Funding}

Agence Nationale de la Recherche (ANR) (ANR-14-CE26-0036 and ANR-15-CE39-0007).

\section{References}

[1] B. Hardy, A. Berrou, S. Guilbaud, M. Raybaut, A. Godard and M. Lefebvre, "Compact, single-frequency, doubly resonant optical parametric oscillator pumped in an achromatic phase-adapted double-pass geometry," Opt. Lett. 36, 678-680 (2011)

[2] M. Raybaut, T. Schmid, A. Godard, A. K. Mohamed, M. Lefebvre, F. Marnas, P. Flamant, A.Bohman, P. Geiser, and P. Kaspersen, "Highenergy single-longitudinal mode nearly diffraction-limited optical parametric source with $3 \mathrm{MHz}$ frequency stability for CO $\mathrm{C}_{2}$ DIAL," Opt. Lett. 34, 2069-2071 (2009)

[3] B. Jacobsson, V. Pasiskevicius, F. Laurell, E. Rotari, V. Smirnov and L. Glebov, "Tunable narrowband optical parametric oscillator using a transversely chirped Bragg grating," Opt. Lett. 34, 449-451 (2009)

[4] P. Blau, S. Pearl, S. Fastig and R. Lavi, "Single-Mode Operation of a Mid-Infrared Optical Parametric Oscillator Using Volume-BraggGrating Cavity Mirrors," IEEE Journal of Quantum Electronics 44, 867-871 (2008)

[5] O.M.Efimov, L.B.Glebov, L.N.Glebova, K.C.Richardson, and V.I.Smirnov, "High-efficiency Bragg gratings in photothermorefractive glass," Appl. Opt. 38, 619-627 (1999) 